\title{
A PSYCHOLINGUISTIC APPROACH TO THE CLASSIFICATION, EVALUATION AND REMEDIATION OF LANGUAGE DISORDER
}

\author{
Ondene van Dulm \\ University of Stellenbosch
}

\section{Introduction}

In recent years there has been a shift in focus in the approach to the classification, assessment and remediation of child and adult language disorder. A psycholinguistic approach has been adopted by many linguists and clinicians. Within the field of psycholinguistics, an attempt is made to interpret the phenomena of both normal and disordered language according to models of language processing. Phenomena of disordered language, then, are interpreted as being due to deficits in the language processing system. The language processing system is considered to be the means by which the speaker-listener expresses himself/herself verbally, and comprehends the expressions of others. A language processing model is a theorist's representation of this system.

Psycholinguistically based research into the language of aphasics has led to advances in the understanding of normal language processing, on the assumption that the phenomena of aphasic speech, as the manifestation of a disturbed language processing system, are directly related to the structure and function of an intact system. In other words, a system that malfunctions in a certain way can do so only if it has a certain structure and function in the first place. As Berndt (1991) notes, "studies of (the syntactic aspects of - OvD) aphasia can contribute to our understanding of how syntactic processes function in the normal speakerlistener".

Traditionally, language-disordered patients have been classified according to their medicallydiagnosed etiology or site of lesion, for example, a Broca's aphasic is someone who has sustained damage to Broca's area. Patients have also been classified according to their symptom complex, for example, a Wernicke's aphasic is someone who exhibits fluent speech with intact function words and syntactic structure but little meaning. 
This traditional approach to the classification of language disordered patients does not focus on each patient as an individual, with a unique deficit in his/her language processing system. Rather, the focus is on grouping patients with similar etiologies and/or similar symptoms. In clinical practice, such groups of patients are then subject to similar assessment and remediation methods, with little attention to the specific needs of individual patients.

Garman (1990) mentions a further shortcoming of such an approach to the classification of language disorder, namely that the focus on the surface manifestation of linguistic impairment (i.e. the symptom) does not allow a hypothesis to be proposed about the causal deficit in the language processing system. Without such insight into the possible underlying deficit, appropriate assessment and remediation are difficult to plan.

The need for and the advantages of a shift in focus from the traditional approach to the classification, assessment and remediation of language disordered patients, to a psycholinguistically based approach, will be discussed below. The limitations of the psycholinguistic approach will then be discussed. First, however, a model of language processing, namely Garrett's (1982) model of sentence production, will be explained.

\section{Garret's (1982) model of sentence production}

According to Harris and Coltheart (1986), a sentence such as The dog chased the cat down the road would be processed as follows by the mechanisms represented by Garrett's model of sentence production (see figure 1 below). In the message level representation is the speaker's prelinguistic idea of the concept(s) he wishes to express. Before the functional level of representation is achieved, the lexicon is searched for the abstract representations of the content words, namely dog, cat, chase and road. A functional predicate-argument structure is then constructed on the basis of the argument information encoded with the verb. The verb (or predicate) specifies the number of arguments and assigns their thematic roles. In this case, the verb chase requires three arguments, namely AGENT (dog), RECIPIENT (cat) and LOCATION (road). The abstract forms of the content words are slotted into this predicateargument structure, yielding the functional level representation of the sentence. At this stage, the mental lexicon is searched for a second time, to retrieve the phonological representations 
of the content words, namely /dpg/, /kæt/, /t $\int \varepsilon$ is/ and /.ıəud/. A planning frame for the sentence is generated, which specifies the form the sentence will take, eg. active or passive voice, present or past tense. Once the form of the sentence has been established, the necessary affixes and function words are generated, in this case the, -ed, the, down and the, and their phonological forms accessed from the mental lexicon. The phonological forms of the content and function words are now slotted into the planning frame, yielding the positional level representation. Phonetic rules are applied to generate the phonetic level representation, and, finally, the articulatory level representation is generated, upon which instructions are sent to the articulators to produce the sentence.

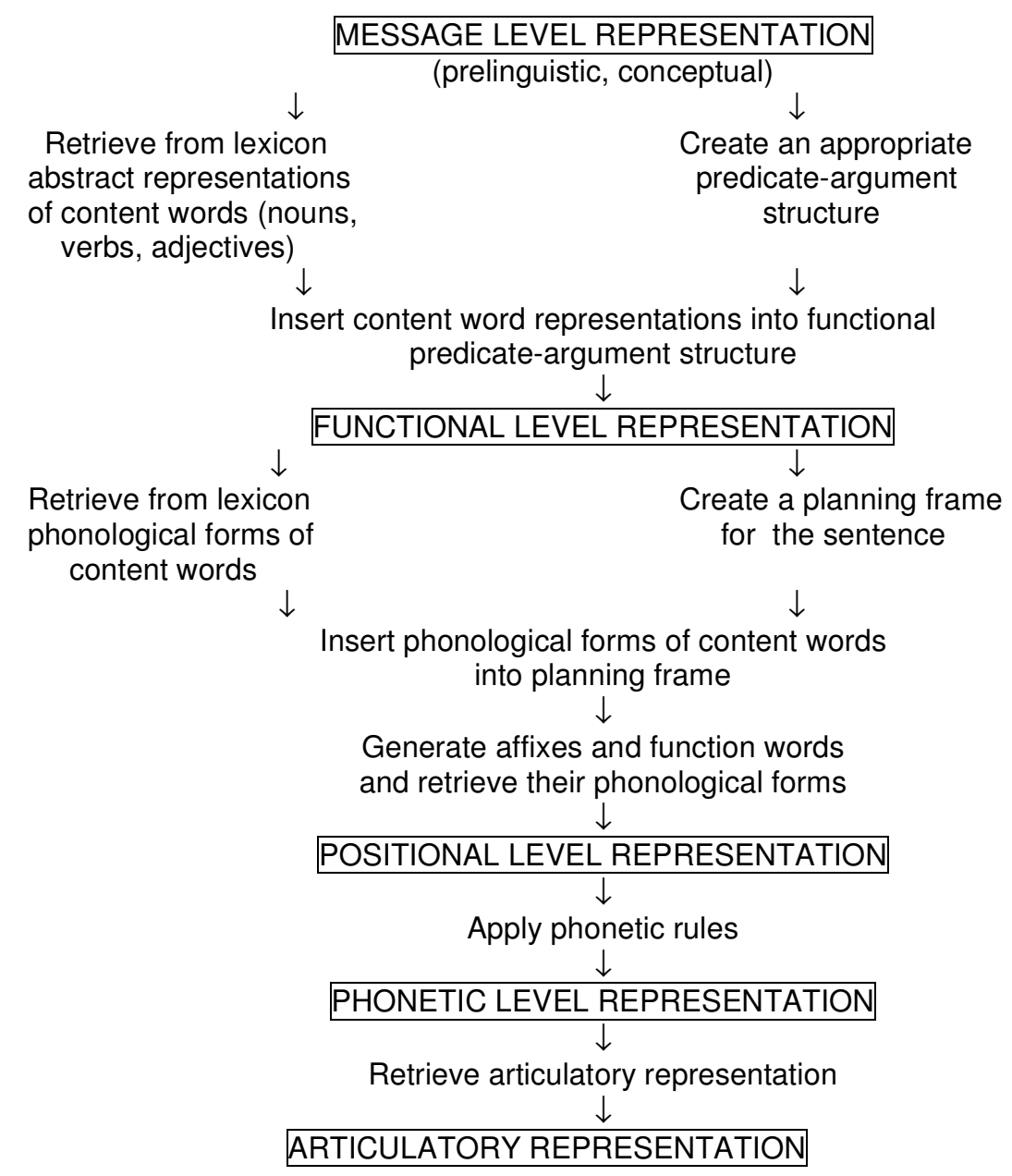

Figure 1: Garrett's (1982) model of sentence production (Harris and Coltheart 1986) 


\section{The need for a shift in focus}

The need for a shift in focus from a traditional to a psycholinguistic approach to the assessment, classification and remediation of adult language disorder is evident from cases such as that discussed by Jones (1986). Jones discusses a traditional remediation procedure for an agrammatic patient, the rationale for which is based on the observation that the patient cannot structure a syntactically correct sentence. This symptom of agrammatism has traditionally been treated by means of a production hierarchy, within which exercises are structured to elicit first single words, then phrases, and, finally, sentences. This traditional treatment approach has had limited success, with poor carry-over into spontaneous speech (Jones 1986). A psycholinguistic approach to the problem, however, suggests the hypothesis of a mapping deficit underlying the symptom of poor sentence construction. On Garrett's (1982) model, this deficit lies at the functional level, in the mapping between semantics and syntax, where lexical items are slotted into the syntactic structure (Jones 1986).

Jones (1986) assessed an agrammatic patient from a psycholinguistic perspective, and devised an individual-specific remediation program to focus on the functional level of sentence processing, and to utilise the patient's intact abilities. Where years of traditional remediation had failed, this psycholinguistically based remediation succeeded. The shift in focus from a traditional to a psycholinguistic perspective entailed a shift in focus from surface symptoms to the underlying deficit (Jones 1986).

A similar need for a shift in focus exists in the field of child language disorder. Fletcher (1990) discusses a traditional assessment and classification tool, the Language Assessment, Remediation and Screening Procedure (LARSP). The LARSP profile, based on categories of normal development, has been used to classify pre-school children as language-delayed and/or language-disordered. It has also been used as a basis for the planning of remediation. Fletcher (1990) reiterates Miller's (1987) cautionary note, that describing child language disorder by using categories of normal language development may allow features unique to each language-disordered child to be overlooked. For example, Fletcher (1990) noted various

error types in the syntax of language-disordered children which could not be reflected 
accurately by the LARSP categories. These errors could more easily be interpreted from a psycholinguistic perspective, as deficits to the language processing system.

Fletcher (1990) encourages a "dynamic view of language production, with a series of levels or components delineated on the route (from the conceptual intention - OvD) to the phonetic string”. Within a psycholinguistic approach, the deficits which possibly underlie the formulation problems of language-disordered children may be interpreted according to Garrett's (1982) model. The errors in syntactic relations noted by Fletcher (1990), such as My mum was take me a picture for My mum was taking a picture of me may occur at the functional level, due to incorrect thematic role application (where me is assigned the incorrect thematic role of BENEFACTIVE, instead of appearing as the complement within the object noun phrase a picture of me). Blends, such as Where shall we put it here? for Shall we put it here? and/or Where shall we put it? (Fletcher 1990) may occur due to competition between different syntactic frames for the same message, i.e. between the conceptual and the functional levels. Lexical selection errors, as in She's sitting on a table instead of She's sitting on a chair, in which the word selected is semantically related to the target item, may occur at the positional level (Fletcher 1990). Viewing child language disorder from a psycholinguistic perspective may, in this way, refine classification, assessment and remediation procedures.

\section{Contributions of a language processing perspective}

A psycholinguistic approach to child and adult language disorder will be justified, and clinicians encouraged to adopt such an approach, if language processing models can be shown to make certain contributions to the clinical process. Specifically, models must (a) allow appropriate classification of language-disordered patients; (b) lead in-depth assessment; (c) suggest the focus of remediation; (d) act as a source of ideas about remediation methods; (e) focus on the individual; and (f) allow for adaptation and refinement of the models themselves. 
The following sections will reveal the extent to which the above goals are met, by offering a survey of the literature on the application of the psycholinguistic approach to child and adult language disorder.

\section{The application of the psycholinguistic approach}

\subsection{The classification of language-disordered patients}

Bishop and Rosenbloom (1987) mention a "growing dissatisfaction" with the traditional medical approach to the classification of language disorders, within which classification is often based on site-of-lesion or etiology, while the underlying deficit is ignored. More recently, a linguistic approach toward classification has been adopted, in which the clinical features of the disorder are taken into account. For example, the patient is classified as a Broca's aphasic if his scores on formal evaluation procedures indicate that his linguistic performance correlates with that of Broca's aphasics in general. It may be that the psycholinguistic approach, which aims to determine the site-of-lesion in the language processing system on the basis of the observed clinical features, will take both etiological and linguistic information into account.

An example of the classification of child language disorder according to the psycholinguistic approach comes from Fletcher (1990), who aimed to interpret the data of a group of languagedisordered children from the perspective of a dynamic model of language processing, namely Garrett's (1982) model. The children were grouped according to the locus of their deficits in the language processing system. One group showed few syntactic errors in production and so could be classified as being in need of remediation focusing on language comprehension. A second group exhibited syntactic problems, such as those in the examples given above, as well as problems in phonological mapping, such as the omission of unstressed syllables and brief phonetic elements. This group could be classified as needing remediation focusing on the functional and positional levels of processing on Garrett's (1982) model. A third group showed only high-level problems, possibly in the mapping between conceptual and functional levels, which led to reformulations and mazes, such as because they get I get scared (Fletcher 1990). 
In this way, Fletcher (1990) demonstrated the use of a language processing model in the classification of child language disorder. Within a traditional classification model, based on observed clinical features and test scores, such children may all have been classified together as simply language-disordered or language-delayed. Within the psycholinguistic classification model, the nature of the underlying processing disorder was considered, and indications for individual-specific remediation were available.

Models of normal language processing have also been used successfully to classify adult aphasics into clinically valid categories. Schwartz (1987), for example, states that the phenomena of aphasic speech can be better described from a psycholinguistic perspective than according to a traditional neuroanatomical (medical) model. Schwartz (1987) uses Garrett's (1982) model to account for various aphasic phenomena, for example for those of Wernicke's aphasia. Garrett's (1982) dual retrieval account of lexical access allows the source of the paraphasias typical of Wernicke's aphasia to be located on the model. Semantic paraphasias arise due to disruption of the first pass through the mental lexicon, when the semantic specification fails to access the relevant lexical item. Phonemic paraphasias arise due to disruption of the second pass through the mental lexicon, when a lexical target accesses a degraded phonological representation. Thus, a psycholinguistic approach allows one to postulate the deficit which causes a certain aphasic phenomenon. Patients with similar deficits can be grouped together. In this way, language processing models aid the classification of language-disordered adults.

\subsection{In-depth assessment}

Chiat and Hirson (1987) present a psycholinguistic case study of a child diagnosed, according to the traditional approach, as a developmental dysphasic, meaning that her linguistic performance was delayed and/or abnormal, in the absence of any observable physical cause. The aim of the in-depth, psycholinguistically based evaluation of the child's spontaneous utterances was to generate a hypothesis about the possible nature of the constraints on the child's mapping from conceptual intention to spoken output. Specifically, the aim was to postulate the locus of the deficit on a language processing model which entails conceptual, syntactic and phonological levels of processing. 
Chiat and Hirson's (1987) case study was based on a psycholinguistic description of the child's language behaviour, i.e. of the relationship between the conceptual intentions she wished to express and the language she used to express these. An aim was to identify patterns in her linguistic mapping from conceptual intention to utterance. Analysis of her spontaneous utterances revealed:

(a) the variety and complexity of conceptual intentions exceeded her ability to map these linguistically;

(b) from a gross syntactic, semantic and phonological point of view, constituent structure was correct;

(c) omissions and substitutions involved syllables and words occurring pre-stress within the prosodic structure of words and sentences, respectively; and

(d) lexical substitutions occurred for modifiers which were semantically and syntactically dependent on the head they modified, and these substituted items were phonologically weak (Chiat and Hirson 1987).

From a psycholinguistic perspective it may be proposed that this child, labelled as a developmental dysphasic, had a problem with word and sentence phonology (Chiat and Hirson 1987). Her errors were not necessarily determined by syntactic factors; rather, omissions and substitutions affected unstressed elements preceding stressed elements in words and sentences. The deficit underlying the child's symptom, namely limited syntactic structure, may have been a constraint on the processing of phonological detail within a rhythmic structure. In the language processing system, the locus of the deficit is at the phonological level of sentence production. Thus, a psycholinguistic approach, in this case, offered a more specific diagnosis than did the traditional approach, and could, in turn, lead to more focused remediation.

In the field of adult or acquired aphasia, a psycholinguistic approach has also been used successfully to lead in-depth assessment. Marshall, Pring and Chiat (1993) advocate an individual-specific evaluation based on language processing theory which follows a step-bystep process to determine the possible locus of deficit in the language processing system. Marshall et al.'s (1993) approach to the assessment of agrammatic patients is based on a 
model of normal language processing, again Garrett's (1982) sentence production model. Garrett's (1982) model allows a hypothesis of a mapping deficit to account for the phenomena of agrammatism, rather than the simple suggestion that agrammatic patients cannot construct grammatical sentences (Marshall et al. 1993).

Marshall et al. (1993) present a patient, MM, whose production was limited to single words, with a striking lack of verbs. In the following step-by-step investigation, each evaluation procedure was motivated by phenomena observed in MM's linguistic performance.

(a) An investigation into verb production revealed that failure to produce verbs could not be attributed to a frequency effect.

(b) An action description task revealed that the problem involved more than just a problem with verb access.

(c) A verb-picture matching task revealed that the verb access problem may have arisen in the semantic system.

(d) Sentence judgement and sentence ordering tasks revealed that the syntactic information encoded with verbs was unavailable.

(e) Event conceptualization tasks revealed difficulty in processing role information.

(f) A sentence comprehension task revealed poor comprehension of role-reversible sentences.

This psycholinguistically motivated evaluation revealed problems with the processing of verbs (Marshall et al. 1993). Verb access was reduced, as was access to syntactic information (eg. argument and thematic role information) encoded with verbs. Psycholinguistically motivated remediation following this investigation would focus on the accessibility of verbs and their associated syntactic information. It is evident from such an example that a psycholinguistic approach to language disorder appears to provide a more satisfactory explanation for certain linguistic phenomena than do the more traditional methods of investigation. 


\subsection{Focus of remediation}

Traditional classification into clinical categories of aphasia, such as Broca's or Wernicke's aphasia, offers little direct guidance for remediation. Patients are categorized according to their symptoms, i.e. the linguistic manifestations of the actual deficit. Basso, Razzano, Faglioni and Zanobio (1990) state that "clinical categories are of limited use as a basis for informed remediation". This is because there is no direct link between a clinical category and a universally effective remediation strategy. Psycholinguistically motivated evaluation, on the other hand, as will be seen below, may offer clearer guidelines for remediation, as the focus is on the deficit in language processing which underlies the symptom.

Nickels, Bing and Black (1991) present an aphasic patient, AER, whose psycholinguistically based evaluation revealed a deficit in retrieving thematic role information and/or a deficit in mapping thematic roles onto syntactic representations. AER's mapping deficit affected both comprehension and production. The psycholinguistically based evaluation led to a two-stage remediation programme.

The first stage focused on comprehension. The aim was to make AER aware of the relationship between the role of a participant in an event, and the position of the noun phrase representing that participant in a sentence. The tasks in the first stage required AER to order visually-presented sentences to describe pairs of pictures. In the pairs, the pictures were identical except in one respect. For example, the identity of the agent of the action would differ, as in The monk writes a letter vs The robber writes a letter (Nickels et al. 1991). AER was encouraged to identify the verb, which determines the thematic roles, in the written choices presented. Further, he had to identify the agent of the action, in order to choose the correct sentence ordering of subject, verb and object, or agent, action and theme.

The second stage of remediation focused on production. AER had established the principles of assigning the roles of agent and theme to the subject and object positions in a sentence, respectively, and was now encouraged to implement these principles in production (Nickels et al. 1991). Using the same pairs of pictures, AER was now required to produce spoken sentences about each picture. If he produced only one phrase in response to a picture, he was 
required to indicate whether it should fill a subject, verb or object position. Initially, AER struggled with verb retrieval, but gradually this improved, as did his production of correctly ordered sentences. After some time, AER even achieved success in correctly ordering rolereversible sentences, such as The boy kicked the girl.

Results of the above remediation revealed that AER could comprehend auditorily-presented reversible sentences in a sentence-picture matching task. In production, the structure of AER's sentences improved significantly, as did his verb retrieval. The psycholinguistic approach to the disorder enabled the researchers to isolate the deficit in AER's language processing system and to design remediation specifically for the deficit, which led to the predicted specific improvements (Nickels et al. 1991). It is possible that the traditional categorization of this patient as agrammatic would not have guided a therapist to such an individual-specific remediation program.

\subsection{Source of ideas about remediation methods}

Language processing models may also act as a source of ideas about remediation methods. A common problem among aphasic patients is word-finding, in, for example, a confrontation naming task. Lesser and Milroy (1993) discuss naming remediation with two distinct groups of patients with naming deficits. The groups were distinguished according to the locus of the deficit in the language processing system. In one group, psycholinguistically based evaluation suggested that a deficit in the phonological output lexicon (POL) led to the naming difficulties. These patients were able to retrieve semantic representations, but needed extra activation of the POL to access the full phonological representation to pronounce the whole word. POL activation was aided by the training of phonemic cueing during remediation.

In a second group of patients, psycholinguistically based evaluation revealed that the deficit in the language processing system causing the naming difficulties could be located either in the semantic system or in the link from the semantic system to the POL. For these patients, reactivation remediation was indicated, to enhance semantic abilities. Such semantic therapy could include, for example, word-picture matching tasks and categorization tasks. It can be 
seen that the application of a psycholinguistic model in the diagnosis of a patient's disorder may offer ideas about appropriate remediation methods (Lesser and Milroy 1991).

Howard, Patterson, Franklin, Orchard-Lisle and Morton (1985) also show how theoretically motivated remediation, based on psycholinguistic theory, may lead to significant improvements in word retrieval. Two techniques are suggested which may be used to treat aphasic patients with naming deficits. Each technique operates at a different level of lexical processing on a language processing model. Semantic techniques require the patient to process the meaning corresponding to a name. Phonological techniques provide the patient with phonological form information to aid retrieval of the name. Thus, approaching a naming deficit from a psycholinguistic perspective may make available a variety of possible remediation methods, each aiming to improve function at a certain level of language processing. Such specific remediation methods may be more beneficial than traditional remediation methods, which did not focus on the deficit in the language processing system.

\subsection{Focus on the individual}

The above examples all show how the psycholinguistic approach to the classification, assessment and remediation of language disorder focuses on the individual patient. A patient is not labelled and treated as one of a group. Rather, each patient is seen as having a unique problem at a particular point in language processing. Ongoing assessment is based on the observed phenomena and the patient's responses to evaluation and remediation tasks.

Hillis (1993), for example, points out that a single treatment strategy may sometimes be appropriate for patients with different deficits in the language processing system, while patients with a common deficit may not always respond equally well to a single treatment strategy. There is no direct one-to-one relationship between locus of deficit and (a) the clustering of features, and (b) effective treatment strategies. The use of an individualised profile, in which language disorder is accounted for within a psycholinguistic framework, is

possibly most appropriate in guiding assessment and remediation under these circumstances. 


\subsection{Adaptation and refinement of models}

The study of language disorder from a psycholinguistic perspective may lead to the refinement of models of normal language processing. As Coltheart, Bates and Castles (1994) state, "the use of data collected in experimental studies of people with impairments (can be used - OvD) as evidence for adjudicating between, testing or developing models of normal cognitive processing”. Such adaptation and refinement of language processing models are necessary, as no conclusion has yet been reached on which of the many models best represents the language processing system.

Within the field of psycholinguistics, for example, there has long been disagreement concerning, among other things, the nature of the semantic system as a component of language processing. One body of opinion argues for the existence of multiple modalityspecific semantic systems, each responsible for processing stimuli in a specific modality, eg. visual, verbal, auditory-non-verbal, tactile, etc. The opposing opinion is that there is a single multimodal semantic system, responsible for all semantic processing.

Shallice (1987) raises three arguments in favour of multiple modality-specific semantic systems. Firstly, the occurrence of cases of modality-specific aphasia points to multiple semantic systems. In modality-specific aphasia, stimuli in all modalities but one, eg. tactile, can be named by the patient. In such a case, the patient can name a picture, or an auditorilydescribed object, but cannot name an object he is exploring tactilely. A possible explanation is that there exist multiple modality-specific semantic representation systems, and there is an impairment in the tactile system, in this case, or in the transmission from the tactile system to the verbal system (Shallice 1987).

Secondly, Shallice (1987) points out the case of a patient, AR, who presented with semantic access dyslexia. In a word-reading task, AR was aided more by a verbal cue than by a picture. Object naming was also difficult for AR. Shallice (1987) offers the explanation that AR could not transmit information between his visual semantic system and his verbal system. Thus, his reading was not aided by a picture, and his picture-naming was not aided by a verbal 
cue. This example points to the possible existence of multiple modality-specific semantic systems.

A third line of evidence for multiple semantic systems, offered by Shallice (1987), comes from the study of aphasic patients who have lost information from their semantic systems. Such patients often show differences in performance between modalities. For example, a patient may be able to describe a visually-presented object, but not the auditorily-presented word. In such a case, the patient's visual semantic system may be intact, while information has been lost from his auditory-verbal semantic system. Evidence of such phenomena may be used to argue in favour of the existence of multiple modality-specific semantic systems.

Evidence does exist, on the other hand, in support of a single multi-modality semantic system. Such a system would be responsible for all semantic processing, regardless of input or output modality. Hillis, Rapp, Romani and Caramazza (1990) mention a patient, KE, who made semantically related errors in various tasks, eg. oral reading, written naming, word-picture matching and naming from tactile input. Analysis of KE's performance revealed similar frequency and type of error across all modalities of input and output (Hillis et al. 1990). This qualitatively and quantitatively similar level of performance across modalities suggests the possibility of damage to a single semantic processing system, responsible for all lexicalsemantic processing. Such damage would result in impairment of processing in all tasks involving the semantic system.

The matter of a single multi-modality semantic system versus multiple modality-specific systems may only be resolved through further research. If conclusive evidence is obtained to support either one hypothesis or the other, language processing models may have to be adapted to correctly represent the structure and function of the semantic component. This is an example of how psycholinguistically oriented investigations into disordered language lead to the adaptation and refinement of models of normal language processing. 


\section{The limitations of the psycholinguistic approach}

The above examples all reveal the clinical benefit of a psycholinguistic approach to child and adult language disorder. However, the approach is limited in its clinical applicability. Coltheart, Bates and Castles (1994) suggest that, where language processing models fail to aid treatment planning, it is because current models are not yet specific enough. For example, a common problem for aphasic patients is a difficulty in semantic processing. Such a problem may manifest as a difficulty in any task requiring access to the semantic system, including picture-naming, naming to a definition, or defining a printed word. The deficit underlying this phenomenon of aphasic language may be in access to the semantic system. If this were so, a language processing model would have to explain exactly how the semantic system is accessed, if it were to aid the planning of remediation. Alternatively, the deficit may involve damage to the semantic representations themselves. In this case, the model would have to explain what the notion of a semantic representation actually entails. No current model offers such explanations (Coltheart et al. 1994). This suggests that further research in the psycholinguistic approach to language disorder is necessary in order to generate more specific models.

Patient QN, described by Coltheart et al. (1994) in a discussion of the above point, presented with, among other things, severely impaired reading. His reading problem was morphological in nature, with the most common errors being omission, addition or alteration of suffixes. A language processing model suggested three possible loci of the deficit, namely (a) within the visual input lexicon, (b) within the central morphological knowledge store, or (c) within the POL. These loci represent quite different impairments, which would require quite different remediation strategies. Psycholinguistically based evaluation was carried out to determine the locus of deficit before remediation could be planned.

A task requiring morphological comprehension was suggested by the language processing model. The task required QN to access the meanings of suffixes (Coltheart et al. 1994). For example, he had to decide that dancer refers to a person, while dancing (morphological foil), danger (visual foil) and ballet (semantic foil) do not. This evaluation revealed that QN's problem specifically involved the recognition of suffixes; the deficit was located in the visual 
input lexicon. The language processing model allowed for the precise identification of a specific impairment in reading, and yet model-based treatment was entirely unsuccessful. A number of treatment methods were attempted, guided by the psycholinguistic perspective, but no improvement in QN's ability to read suffixed words was observed (Coltheart 1994). This example illustrates a limitation of the psycholingusitic approach to language disorder, namely that there is often no direct link between the theoretical interpretation of a disorder and an effective remediation strategy.

It has become apparent in the previous sections that a psyholingusitic perspective on language disorder has a lot to offer in aid of devising assessment and remediation procedures. However, language processing models have inherent limitations, and much refinement is necessary before they alone can shape the clinical process. Chiat and Jones (1988) highlight some limitations of current language processing models. Firstly, many phenomena of disordered language cannot be explained by the models, which are, as yet, too simplistic. For example, Lesser and Milroy (1993) mention features of deep dyslexia which could not be accounted for by the language processing model used in their study, which included a phonological analysis component, a phonological input lexicon, a semantic system, a graphemic output lexicon and a graphemic buffer. The occurrence of visual errors, eg. single for signal, and errors of derivational morphology, eg. editor for edition, could not be accounted for in terms of the model. Such a model offers no explanantion for the dyslexic features, and so would be of little use in planning assessment and remediation.

Secondly, Chiat and Jones (1988) suggest that it should not be assumed that the function of the impaired language processing system is identical to that of the normal system, with merely local modification at an impaired component. Rather, it may be that brain damage causes processing that is qualitatively different from normal, due to the adaptation of one component to compensate for the malfunction of another. There may be knock-on effects, i.e. automatic adaptation of the impaired system to exploit connections and intact functions in novel ways. For example, the phenomena of agrammatism, namely poor syntactic structure and the omission of function words, may be explained by such adaptation of an impaired system. Berndt (1991) suggests that the aphasic patient, faced with the likelihood of misarticulating a sentence, may omit unessential items (eg. function words) and/or may 
simplify sentence structure. In this case, a possible phonological processing deficit gives rise to the symptoms of agrammatism. Once again, it is evident that, although models of normal language processing offer much insight into language disorder, caution must be exercised when applying a psycholinguistic approach to assessment and remediation, as the phenomena of disordered language may go beyond that which can be accounted for by the models.

A third limitation of the psycholinguistic approach to language disorder is that it is not always clear that one can label a clinical procedure as being psycholinguistic in nature. Hillis (1993) states that it is not always clear, in clinical studies, how a language processing model has contributed to the development of remediation strategies. Further, it is often not clear that the remediation based on models is any different from traditional remediation. The contribution of a psycholinguistic approach is perhaps restricted to offering a means of cognitive analysis of a patient's language behaviour. Remediation can then be based on a clinician's intuitions about what may improve the function of a certain component in the language processing system, or what may help the patient by-pass the faulty component by utilizing intact mechanisms (Hillis 1993).

It may be that the limitations in the role of language processing models in the clinical process go beyond the fact that current models are simplistic, as Coltheart (1994) and Chiat and Jones (1988) suggest. Hillis and Caramazza (1994) suggest that, even if more detailed models were developed, there remains the issue of whether and how damaged components in the language processing system can recover. Language processing models may suggest how language is processed at various levels, but they do not suggest how recovery of function can occur following brain damage. This issue could only be resolved by consideration of the neurology of the system, and how stimulation may change the damaged system. "A model of cognitive theory may be a NECESSARY component of a theory of rehabilitaion, but it is not SUFFICIENT" (Hillis and Caramazza 1994). 


\section{Conclusion}

It has been described how the psycholinguistic approach to the classification, assessment and remediation of child and adult language disorder arose in the face of various shortcomings of the traditional approach. Examples of contributions made by language processing models to the field of language disorder have been discussed. The psycholinguistic perspective appears to have a definite role to play in the clinical process, its emphasis on the individual being its

prime contribution. Further, the application of language processing models in the field of language disorder leads to the essential adaptation and refinement of models of normal language processing.

Limitations of models in shaping the clinical process have also been discussed. Models are not yet detailed enough to aid the planning of all remediation, as some phenomena can still not be explained in terms of the models. A direct link between models of normal language processing and disordered language has yet to be established. Thus, while a psycholinguistic approach may begin to overcome some of the problems related to the traditional approach, it is not, as yet, the ideal solution. Clinicians would do well to utilize language processing models to their full potential, while remaining aware of the limitations of the psycholinguistic approach to language disorder. 


\section{REFERENCES}

Basso, A., G. Razzano, P. Faglioni and M.E. Zanobio. 1990. Confrontation naming, picture description and action naming in aphasic patients. Aphasiology 4(2): 185-195.

Berndt, R.S. 1991. Sentence processing in aphasia. In M.T. Sarno (ed). Acquired aphasia. New York: Academic Press.

Bishop, C.V.M. and L. Rosenbloom. 1987. Classification of childhood language disorders. In W. Yule and M. Rutter (eds). Language development and disorders. London: McKeith Press.

Buckingham, H.W. 1981. Lexical and semantic aspects of aphasia. In M.T. Sarno (ed). Acquired aphasia. New York: Academic Press.

Chiat, S. and A. Hirson. 1987. From conceptual intention to utterance: a study of impaired language output in a child with developmental dysphasia. British journal of disorders of communication 22: 37-64.

Chiat, S. and E. Jones. 1988. Processing language breakdown. In M. Ball (ed). Theoretical linguistics and disordered language. London: Croom-Helm.

Coltheart, M., A. Bates, and M Castles. 1994. Cognitive neuropsychology and rehabilitation. In M.J. Riddoch and G.W. Humphreys (eds). Cognitive neuropsychology and cognitive rehabilitation. London: Lawrence Erlbaum Asssociates.

Fletcher, P. 1990. Evidence from syntax for language impairment. In J. Miller (ed). Research on child language disorders: a decade of progress. San Diego: College Hill Press.

Garman, M. 1990. Psycholinguistics. Cambridge: Cambridge University Press. 
Harris, M. and M. Coltheart. 1986. Language processing in children and adults: an introduction. London: Routledge.

Hillis, A.E. 1993. The role of models of language processing in rehabilitation of language impairments. Aphasiology 7(1): 5-26.

Hillis, A.E. and A. Caramazza. 1994. Theories of lexical processing and rehabilitation of lexical deficits. In M.J. Riddoch and G.W. Humphreys (eds). Cognitive neuropsychology and cognitive rehabilitation. London: Lawrence Erlbaum Asssociates.

Hillis, A.E., B. Rapp, C. Romani and A. Caramazza. 1990. Selective impairments of semantics in lexical processing. Cognitive neuropsychology 7(3): 191-243.

Howard, D., K. Patterson, S. Franklin, V. Orchard-Lisle and J. Morton. 1985. Treatment of word retrieval deficits in aphasia. Brain 108: 817-829.

Jones, E.V. 1986. Building the foundations for sentence production in a non-fluent aphasic. British journal of disorders of communication 21: 63-82.

Lesser, R. and L. Milroy. 1993. Linguistics and aphasia. London: Longman.

Levelt, W.J.M. 1992. Lexical access in sentence production. Cambridge: Blackwell.

Marshall, J., T. Pring and S. Chiat. 1993. Sentence processing remediation: working at the level of the event. Aphasiology 7(2): 177-199.

Nickels, L., S. Bing and M. Black. 1991. Sentence processing deficits: a replication of remediation. British journal of disorders of communication 26: 175-199. 
Schwartz, M.F. 1987. Patterns of speech production deficit within and across aphasia syndromes: application of a psycholinguistic model. In M. Coltheart, R. Job and G. Sartori (eds). The cognitive neuropsychology of language. London: Lawrence Erlbaum Associates.

Shallice, T. 1987. Impairments of semantic processing: multiple dissociations. In $\mathrm{M}$. Coltheart, R. Job and G. Sartori (eds). The cognitive neuropsychology of language. London: Lawrence Erlbaum Associates. 